\title{
Ulcerative colitis complicated by renal cell carcinoma: a series of three patients
}

\author{
J Satsangi, J Marshall, D Roskell, D Jewell
}

\begin{abstract}
Three patients are described in whom renal cell carcinoma was diagnosed between seven months and 14 years after the onset of ulcerative colitis. All three had required treatment with corticosteroids and azathioprine, as well as maintenance therapy with 5-aminosalicylate derivatives, for chronic active colitis. One patient had coexisting polymyalgia rheumatica; one had primary biliary cirrhosis. It is uncertain whether this association, which has not previously been described, has pathogenetic importance or has arisen simply by chance. Genetic predisposition or the effect of drug treatment, particularly immunosuppression, may be pertinent to the co-occurrence of these diseases.

(Gut 1996; 38: 148-150)
\end{abstract}

Keywords: ulcerative colitis, renal cell carcinoma, immunosuppression.

\section{Case history}

\section{PATIENT 1}

A 62 year old male, white, farm storeman presented as an emergency in July 1984 with a 48 hour history of left loin pain and nausea. Ulcerative colitis had been diagnosed 14 years previously and had had a chronic relapsing course, requiring continuous maintenance therapy with sulphasalazine ( $1 \mathrm{~g}$ twice daily) and intermittent courses of oral and topical steroids. Between 1980 and 1984, therapy with azathioprine $(2 \mathrm{mg} / \mathrm{kg} / \mathrm{d})$ had maintained remission. Blood tests, including a full blood count, had been monitored regularly. Flexible sigmoidoscopy in 1982 had shown quiescent colitis confined to the rectum and distal sigmoid colon. The patient had smoked 20 cigarettes a day since the age of 18 years.

On physical examination, he was found to be hypertensive $(180 / 110 \mathrm{~mm} \mathrm{Hg})$ and overweight (91 kg). A mass was palpable in the left loin. Dip-stick urine testing showed microscopic haematuria. Full blood count and all biochemical tests were normal. An intravenous pyleogram showed complete left pelvi-ureteric obstruction. Abdominal ultrasound confirmed a large solid mass in the upper pole of the left kidney. Chest $x$ ray showed no metastasis.
At operation, a round tumour $(6.5 \mathrm{~cm}$ in diameter) was noted in the upper pole of the left kidney. The renal capsule was breached by tumour in one place. No evidence of venous or lymph node spread was present. Left radical nephrectomy was performed. The immediate postoperative recovery was complicated by a relapse of ulcerative colitis that required treatment with intravenous steroids. Histological examination of the tumour confirmed a diagnosis of renal cell carcinoma. The cytoplasm of the malignant cells had a granular eosinophilic appearance, consistent with the oncocytic variant.

Further relapses of colitis were treated with corticosteroids in 1985 and 1986. Azathioprine treatment was reinstituted in 1986 for 18 months but was stopped in 1987. Extensive investigation for pyrexia of unknown origin in 1986 resulted in a diagnosis of polymyalgia rheumatica. In 1994, the patient remained well off all medication and showed no evidence of active colitis or metastatic disease.

PATIENT 2

A 48 year old white housewife presented in June 1993 with a two month history of progressive incapacitating diarrhoea, passing up to 12 bloody motions over 24 hours. She also described lower abdominal pain, anorexia, and one stone weight loss. No features in her medical history pointed to an infective cause: stool cultures had twice been negative in preceding weeks. Hypertension had been diagnosed by the general practitioner 12 months before, and treated with atenolol. She smoked 10-15 cigarettes a day.

On examination, the patient was unwell, febrile $\left(37.5^{\circ} \mathrm{C}\right)$, and dehydrated. Her blood pressure was $150 / 90 \mathrm{~mm} \mathrm{Hg}$ and her weight was $77.3 \mathrm{~kg}$. Abdominal examination showed left sided tenderness. At sigmoidoscopy, the mucosa looked acutely inflamed, granular, and friable. A provisional diagnosis of acute severe ulcerative colitis (confirmed on subsequent histology) was made, and hospital admission was arranged for treatment. Plain abdominal $x$ ray showed mucosal oedema of the left colon and distal transverse colon. Investigation revealed hypokalaemia $(2.8 \mathrm{mmol} / \mathrm{l})$, and liver dysfunction-serum bilirubin was $8 \mu \mathrm{mol} / 1$ (normal range 0-18 $\mu \mathrm{mol} / 10$, alkaline phosphatase activity was $368 \mathrm{U} / 1$ (normal range 
80-250 U/l), albumin was $44 \mathrm{~g} / 100 \mathrm{ml}$, and aspartate aminotransferase activity (AST) $22 \mathrm{U} / 1$ (normal range 10-45 U/1).

Treatment with parenteral fluids and corticosteroids induced remission, and the patient was discharged home. Medication on hospital discharge was oral corticosteroids, olsalazine $500 \mathrm{mg}$ twice daily, and atenolol.

At follow up in September 1993 a relapse in symptoms was evident and oral steroids were restarted together with azathioprine ( $3 \mathrm{mg} / \mathrm{kg} / \mathrm{d}$ ). Azathioprine was stopped after two weeks because of disabling nausea and vomiting which necessitated hospital admission for 48 hours. In December 1993, remission of colitis had been achieved but further investigations of her persistently abnormal liver blood tests were undertaken. Immunology showed a raised titre of antinuclear antibody (1/320) and of anti-mitochondrial antibody (1/160), and normal DNA binding. These features were felt to be most consistent with a diagnosis of primary biliary cirrhosis.

Abdominal ultrasound was performed in January 1994. The liver and common bile ducts looked normal; a solitary gall stone was noted in the gall bladder. However, a heterogeneous mass $(6 \times 6 \mathrm{~cm})$ was present in the upper pole of the right kidney. Computed tomogram showed no evidence for spread into the inferior vena cava (Figure).

Right nephrectomy and needle liver biopsy were carried out in April 1994. No evidence for local spread of tumour was noted. Dramatic haemorrhage from the renal bed necessitated an urgent re-exploration within 12 hours. The history of the resected tumour showed features of clear cell adenocarcinoma. A full recovery was made subsequently. Colonoscopy showed evidence for mild total colitis; endoscopic retrograde cholangiopancreaticography (ERCP) was normal in September 1994.

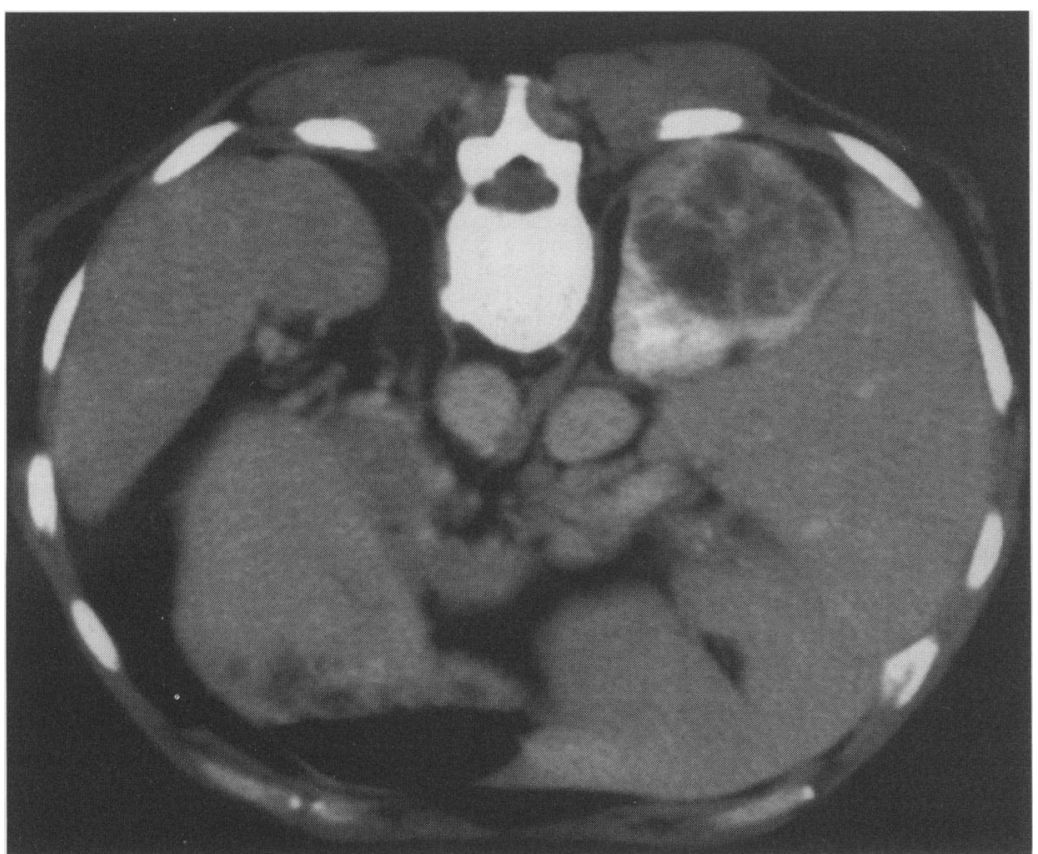

Right renal cell carcinoma.
PATIENT 3

A 55 year old white male accountant, who had smoked 10 cigarettes a day for 35 years, initially presented acutely unwell in July 1989 with high fevers and rigors. Blood culture grew Escherichia coli and mixed anaerobes. The patient was treated with intravenous antibiotics and made a complete recovery. Extensive investigation to determine the source of the sepsis was subsequently performed. Urine culture showed no growth. Abdominal ultrasound showed multiple hepatic cysts, up to $5 \mathrm{~cm}$ diameter. Aspiration revealed clear fluid, sterile to culture. Barium enema showed extensive sigmoid diverticular disease but no other pathology. However, flexible sigmoidoscopy showed confluent inflammation affecting the mucosa of the sigmoid colon and rectum. Histology confirmed chronic ulcerative colitis. Maintenance therapy with sulphasalazine ( $1 \mathrm{~g}$ twice daily) was introduced. The patient remained well until February 1993 when he presented with symptoms of acute colitis, passing up to 10 bloody motions over 24 hours.

On examination, he was obese and overweight $(101 \mathrm{~kg})$. The blood pressure was $180 / 100 \mathrm{~mm} \mathrm{Hg}$. Abdominal examination showed a large irregular liver. Sigmoidoscopy confirmed acute colitis. Blood tests demonstrated a microcytic anaemia (haemoglobin concentration $11.4 \mathrm{~kg} / 100 \mathrm{ml}$ ), raised inflammatory markers (C-reactive protein concentration $115 \mathrm{mg} / 100 \mathrm{ml}$; erythrocyte sedimentation rate $42 \mathrm{~mm} / \mathrm{h}$ ), and normal liver and renal functions.

Topical therapy was ineffective and oral prednisolone and azathioprine $(1.5 \mathrm{mg} / \mathrm{kg} / \mathrm{d}$, commenced in January 1993) were needed to induce remission. Barium enema showed severe active colitis, now affecting the colon distal to the hepatic flexure. Ultrasound demonstrated the known hepatic cysts but normal kidneys. Remission was achieved with difficulty, and the patient remained asymptomatic until October 1993. Medications included: ranitidine ( $150 \mathrm{mg}$ twice daily) sulphasalazine ( $1 \mathrm{~g}$ twice daily) captopril $(50 \mathrm{mg}$ three times daily) and azathioprine $(150 \mathrm{mg}$ once daily).

In October, abdominal ultrasound (performed because of increased abdominal distension) demonstrated a $5 \cdot 2 \mathrm{~cm}$ diameter solid tumour of the lower pole of the right kidney. Computed tomogram showed no local venous or lymphatic spread. Chest $x$ ray appearances were normal.

Right nephrectomy was performed in January 1994. A well circumscribed tumour $(5 \times 4.5 \times 3 \mathrm{~cm})$ was present in the lower pole, having breached the renal capsule in one area. Histology confirmed papillary cell carcinoma of the kidney involving the perinephric fat. The patient's postoperative recovery was rapid; adjuvant radiotherapy was considered to be of uncertain benefit and was not given. In November 1994, a persistent dry cough led to further investigation. Chest $x$ ray and computed tomography showed multiple pulmonary nodules. 


\section{Discussion}

Renal cell carcinoma is a relatively uncommon tumour, accounting for approximately $3 \%$ of primary malignancies. In Oxfordshire in 1990, the incidences were $9.6 \times 10^{-5}$ in men and $4.8 \times 10^{-5}$ women (personal communication, Oxford Cancer Epidemiology Unit). This tumour has not previously been reported as a complication of ulcerative colitis. It is possible that the co-occurrence of these diseases in the patients described may have arisen by chance. All three patients each had individual risk factors for carcinoma of the kidney identified in previous studies ${ }^{1-3}$ (obesity, smoking, hypertension). The association may have pathogenic significance: genetic factors or the effects of drug therapy (particularly immunosuppression) may be pertinent.

There is strong evidence from studies of familial inflammatory bowel disease that genetic predisposition is important in susceptibility to both Crohn's disease and ulcerative colitis. It seems probable that ulcerative colitis does not have a simple Mendelian pattern of inheritance but that a number of distinct genetic loci are involved. The identity of the susceptibility genes is unknown. Although recent evidence implicates HLA class II genes, ${ }^{4}$ detailed linkage analysis studies are required in ulcerative colitis. Cytogenetic observations, linkage analysis, and physical mapping studies have all implicated inactivation of tumour suppressor genes on the short arm of chromosome 3 in the pathogenesis of sporadic as well as familial renal carcinoma. ${ }^{5}$ Therefore, this region may provide candidate susceptibility loci for further studies in ulcerative colitis.

The possible relevance of drug therapy for ulcerative colitis to the development of renal cell carcinoma merits discussion. Each patient had required immunosuppression with systemic steroids and azathioprine in addition to maintenance therapy with sulphasalazine (two patients) or olsalazine. Although impairment of renal function (most commonly caused by interstitial nephritis) has been described in patients taking sulphasalazine ${ }^{6}$ or mesalazine, ${ }^{7}$ no reports of neoplasia complicating maintenance therapy with these drugs have been made. It is of potential relevance that phenacetin, a drug with considerable structural homology to 5-aminosalicylic acid, has been implicated in the pathogenesis not only of interstitial nephritis but also renal cell carcinoma. ${ }^{3}$ Even so, considerable experience with 5-ASA derivatives, especially sulphasalazine, in maintenance therapy of inflammatory bowel disease has accumulated: with this in mind, it seems unlikely that these drugs are involved in the pathogenesis of the neoplasms reported here.

There is considerable concern regarding the prevalence of malignancy in patients treated with long term immunosuppression (in particular azathioprine) either for chronic inflammatory disease or after transplantation. In the present series, one patient had received only two weeks' treatment with azathioprine and it seems unlikely that the drug was involved in tumourigenesis. The other patients had received the drug for 10 months and four years respectively before the diagnosis of renal carcinoma. Connell et $a l^{8}$ have recently reviewed the experience at St Mark's Hospital concerning 755 patients with inflammatory bowel disease treated with azathioprine for between two days and 15 years. Thirty one developed cancer before the age of 85 years, compared with 24.3 expected from rates in the general population (observed:expected ratio $=1 \cdot 27$, $p=0 \cdot 186)$. Colorectal cancers were more frequent than expected but this difference was attributable to chronic extensive colitis, not drug treatment. No cases of renal cell carcinoma were detected: however, given the relative rarity of this tumour, the study may not have been sufficiently powerful to detect an increase in prevalence.

There is very considerable experience of tumours after long term immunosuppression subsequent to organ transplantation, particularly in renal units. Squamous cell carcinoma of the skin and non-Hodgkin's lymphoma are the most frequent neoplasms recorded. ${ }^{9}$ Carcinoma in native kidney ${ }^{10}$ and dysplasia of the epithelial tissues of a graft kidney ${ }^{11}$ have been recorded. However, renal carcinoma is also a recognised complication in the kidneys of patients with chronic renal failure undergoing haemodialysis, in the absence of any immunosuppression: the relevance of azathioprine in the development of these tumours remains to be proved.

The present report has documented the development of renal cell carcinoma in three patients with ulcerative colitis who had required immunosuppressive therapy. The importance of this co-occurrence is uncertain: the importance of genetic factors may be clarified by cytogenetics or linkage analysis. There is, at present, no definite evidence to implicate drug therapy for ulcerative colitis in the development of these renal tumours.

1 McLauglin JK, Mandel JS, Blot WJ, Schuman LM, Mehl ES, Fraumeni JF. A population-based case control study of renal cell carcinoma. $₹$ Natl Cancer Inst 1984; 72: of renal 84 .

2 Yu MC, Mack TN, Hanisch R, Cicioni C, Henderson BE. Cigarette smoking, obesity, diuretic use and coffee consumption as risk factors for renal cell carcinoma f Natl Cancer Inst 1986; 77: 351-6.

3 Lineham WM, Shipley WU, Parkin DR. Cancer of the kidney and ureter in cancer. In: de Vita VT, Hellman S, Rosenberg SA, eds. Principles and practice of oncology. Philadelphia: JB Lippincott Co, 1993: 1023-51.

4 Satsangi J, Jewell DP, Rosenberg WMC, Bell JI. Genetics of inflammatory bowel disease. Gut 1994; 35: 696-700.

5 La Forgia S, Lasato J, Latif F. Detailed genetic and physical map of the $3 p$ chromosome region surrounding the familial renal cell carcinome region surrounding the $t(3,8)$ (p14.2; 124.1). Cancer Res 1993; 53: 3118-24.

t $(3,8)$ (p14.2; 124.1). Cancer Res 1993; 53: 3118-24. induced renal failure. Gut 1992; 33: 1006-7.

7 Thulurath PJ, Ninkovic M, Calam J, Anderson M. Mesalazine induced interstitial nephritis. Gut 1994; 35: 1493-6.

8 Connell WR, Kamm MA, Dickson M, Balkwill AM, Ritchie JK, Lennard-Jones JE. Long-term neoplasia risk after JK, Lennard-Jones JE. Long-term neoplasia risk after azathioprine treatment in in

9 Hoover R, Fraumeni JF. Risk of cancer in renal-transplant recipients. Lancet 1973; ii: 55-7.

10 Lien YH, Kam I, Shanley PF, Schroter GP. Metastatic renal cell carcinoma associated with acquired cystic kidney disease 15 years after successful renal transplantation. Am 于 Kidney Dis 1991; 18: 711-5.

11 Mittal BV, Cotton RE. Severely atypical changes in renal epithelium in biopsy and graft nephrectomy specimens in two cases of cadaver renal transplantation. Histopathology 1987; 11: 833-41. 